\title{
Effects of dietary arginine on inflammatory mediator and receptor of advanced glycation endproducts (RAGE) expression in rats with streptozotocin-induced type 2 diabetes
}

\author{
Man-Hui Pai ${ }^{1}$, Kuan-Hsun Huang ${ }^{2}$, Ching-Hsiang $\mathrm{Wu}^{1}$ and Sung-Ling $\mathrm{Yeh}^{2} *$ \\ ${ }^{1}$ Department of Anatomy, Taipei Medical University, Taipei, Taiwan \\ ${ }^{2}$ School of Nutrition and Health Sciences, Taipei Medical University, Taipei, Taiwan \\ (Received 14 October 2009 - Revised 15 December 2009 - Accepted 2 March 2010 - First published online 14 April 2010)
}

Arginine (Arg) is known to possess numerous useful physiological properties and have immunomodulatory effects. In vitro studies reported that Arg inhibits advanced glycation endproduct (AGE) formation; however, the effects of Arg on the receptor of AGE (RAGE) expression in inflammatory conditions are not clear. The present study investigated the effects of dietary Arg supplementation on inflammatory mediator production and RAGE expression in type 2 diabetic rats. There were one normal control (NC) group and two diabetic groups in the present study. Rats in the NC group were fed with a regular chow diet. One diabetic group (DM) was fed a common semi-purified diet while the other diabetic group received a diet in which part of the casein was replaced by Arg (DM-Arg) for 8 weeks. Diabetes was induced by an intraperitoneal injection of nicotinamide followed by streptozotocin. Rats with blood glucose levels exceeding $1800 \mathrm{mg} / \mathrm{l}$ were considered diabetic. Blood samples and the liver and lungs of the animals were collected at the end of the study for further analysis. Results showed that plasma glucose and fructosamine contents were significantly higher in the diabetic groups than those in the NC group. The DM group had higher fructosamine and C-reactive protein than the DM-Arg group. Immunohistochemical staining showed that the expressions of RAGE in liver and lung tissues were significantly lower in the DM-Arg group than in the DM group. These results suggest that supplemental dietary Arg can decrease AGE-RAGE interactions and consequently reduce tissue damage in rats with type 2 diabetes.

Arginine: Type 2 diabetes: Fructosamine: C-reactive protein: Receptor of advanced glycation endproducts (RAGE) expression

Diabetes mellitus was the fifth leading cause of death in Taiwan in $2008^{(1)}$. Among all diabetic subjects, type 2 diabetes may account for about $90-95 \%$ of the diagnosed patients. Diabetes mellitus is a metabolic disorder characterised by chronic hyperglycaemia and the development of vascular diseases. Diabetic complications appear to be multifactorial in origin. Advanced glycated endproducts, which elicit a wide range of cell-mediated response, are believed to play a central role in these disorders ${ }^{(2)}$. Advanced glycation endproducts are a complex group of compounds formed via non-enzymic reactions between reducing sugars and amine residues on protein, lipids and nucleic acids. Advanced glycation endproduct formation proceeds slowly under normal ambient sugar concentrations, but is accelerated in the presence of hyperglycaemia ${ }^{(2,3)}$. Advanced glycation endproducts can exert their biological effects through receptor-mediated mechanisms, the most important of which is the receptor of advanced glycation endproducts (RAGE). RAGE is a multi-ligand receptor of cell surface molecules which acts as a counter-receptor for diverse molecules ${ }^{(4)}$. Binding of advanced glycation endproducts to the RAGE activates a number of pathways implicated in chronic inflammation and the development of diabetic complications $^{(4)}$.

Arginine (Arg) is a non-essential amino acid for healthy adults. It has been shown to possess numerous physiological properties. Previous studies have demonstrated that Arg supplementation decreases inflammation ${ }^{(5)}$ and improves immune function ${ }^{(6-8)}$. Currently, Arg is added to enteral formulas in an attempt to modulate immune function and improve clinical outcomes of critically ill patients ${ }^{(9)}$. A recent study by Martina et al. ${ }^{(10)}$ showed that Arg plus $\mathrm{N}$-acetylcysteine (a substance that increases NO availability) administration improves endothelial function and reduces inflammatory markers in hypertensive patients with type 2 diabetes. A study by Lucotti et al. ${ }^{(11)}$ also found beneficial effects of oral Arg supplementation in reducing plasma advanced glycation endproduct levels in type 2 diabetic patients. However, the effects of Arg on advanced glycation endproduct-RAGE interactions and subsequent inflammatory reactions in diabetes are not clear. Therefore, we designed the present study to investigate the effects of supplemental

Abbreviations: Arg, arginine; CRP, C-reactive protein; DM, diabetes without arginine; DM-Arg, diabetes with arginine; MCP-1, monocyte chemotactic protein-1;

NBT, nitroblue tetrazolium; NC, normal control; RAGE, receptor of advanced glycation endproducts.

* Corresponding author: Dr Sung-Ling Yeh, email sangling@tmu.edu.tw 
Table 2. Plasma glucose, TAG, total cholesterol, HDL-cholesterol and LDL-cholesterol concentrations and fructosamine content of the groups at the end of the experiment*

(Mean values and standard deviations)

\begin{tabular}{|c|c|c|c|c|c|c|}
\hline \multirow[t]{2}{*}{ Group... } & \multicolumn{2}{|c|}{ NC } & \multicolumn{2}{|c|}{ DM } & \multicolumn{2}{|c|}{ DM-Arg } \\
\hline & Mean & SD & Mean & SD & Mean & SD \\
\hline Glucose (mg/l) & $1235^{\mathrm{a}}$ & 81 & $3047^{\mathrm{b}}$ & 1236 & $2630^{\mathrm{b}}$ & 831 \\
\hline Fructosamine (change in absorbance/min) & $0.11^{\mathrm{a}}$ & 0.01 & $0.19^{b}$ & 0.02 & $0.16^{\mathrm{c}}$ & 0.01 \\
\hline TAG (mg/l) & $520^{\mathrm{a}}$ & 150 & $659^{\mathrm{b}}$ & 167 & $513^{\mathrm{a}}$ & 173 \\
\hline Total cholesterol (mg/l) & $608^{a}$ & 81 & $874^{\mathrm{b}}$ & 130 & $782^{\mathrm{b}}$ & 143 \\
\hline HDL-cholesterol (mg/l) & $124^{\mathrm{a}}$ & 31 & $209^{b}$ & 35 & $200^{\mathrm{b}}$ & 47 \\
\hline LDL-cholesterol (mg/l) & $392^{\mathrm{a}}$ & 84 & $536^{\mathrm{b}}$ & 106 & $506^{\mathrm{b}}$ & 119 \\
\hline
\end{tabular}

NC, normal control; DM, diabetes without arginine; DM-Arg, diabetes with arginine.

${ }_{a, b, c}$ Mean values within a row with unlike superscript letters were significantly different $(P<0.05)$.

* Different treatments were analysed by one-way ANOVA using Duncan's test.

(diluted 1:300; Chemicon, Temecula, CA, USA) for $1 \mathrm{~h}$ at room temperature. After reacting with the peroxidase-linked avidin-biotin complex (Vector, Burlingame, CA, USA) for $1 \mathrm{~h}$ at room temperature, a diaminobenzidine solution kit (Vector) was used to detect RAGE immunoreactivity. Haematoxylin (Sigma) nuclear staining was also applied to contrast the cell nucleus and cytoplasm. All tissue sections were covered with a coverslip by Permount (Fisher Scientific), and measured by using a digital image analysis system (Image Pro Plus 5.1; Media Cybernetics, Silver Spring, MD, USA) after the images were captured on a Zeiss Axiphot light microscope (Carl Zeiss, Jena, Germany) equipped with a $20 \times$ objective lens and a Nikon D1X digital camera (Tokyo, Japan). We used the 'count/size' and 'area' commands to determine the intensity of the RAGE immunoreactivity. The automatic object counting and measuring processes were used to quantify the immunoreactive areas in the sections. Values were expressed as $\mu \mathrm{m}^{2}$. At least ten microscopic fields per section and three independent samples for each group were analysed and the averaged areas were obtained for each group.

\section{Statistical analysis}

All data are expressed as the mean values and standard deviations. Differences among groups were analysed by ANOVA using Duncan's test. $P<0.05$ was considered statistically significant.

\section{Results}

There were no differences in the initial body weights, food intake or weight after feeding the diets for 8 weeks between the two diabetic groups (data not shown).

\section{Plasma glucose and lipids}

Baseline plasma glucose levels were significantly higher in the diabetic groups than those in the NC group. There were no differences in glucose levels between the DM and DM-Arg groups (DM group 2072 (SD 448) mg/l; DM-Arg group 2120 (SD 500) mg/l $v$. NC group 1007 (SD 78) mg/l; $P<0.05)$. The plasma glucose, total cholesterol, HDL-cholesterol and LDL-cholesterol levels in the diabetic groups were significantly higher than those in the NC group. There were no differences in these parameters between the two diabetic groups at the end of the study (Table 2).

\section{Plasma inflammatory mediators}

Plasma CRP, MCP-1 and $\mathrm{PGE}_{2}$ levels were significantly higher in the two diabetic groups compared with the NC group. There were no differences in $\mathrm{MCP}-1$ or $\mathrm{PGE}_{2}$ between the two diabetic groups. However, the CRP level in the DM-Arg group was significantly lower than that in the DM group (Table 3).

Table 3. Plasma concentrations of intercellular adhesion molecule-1 (ICAM-1), monocyte chemotactic protein-1 (MCP-1), C-reactive protein (CRP), IL-6 and $\mathrm{PGE}_{2}$ in the various groups at the end of the experiment*

(Mean values and standard deviations)

\begin{tabular}{|c|c|c|c|c|c|c|}
\hline \multirow[t]{2}{*}{ Group... } & \multicolumn{2}{|c|}{ NC } & \multicolumn{2}{|c|}{ DM } & \multicolumn{2}{|c|}{ DM-Arg } \\
\hline & Mean & SD & Mean & SD & Mean & SD \\
\hline ICAM-1 (ng/ml) & $25 \cdot 9$ & 3.3 & 30.9 & $7 \cdot 1$ & 31.9 & 8.7 \\
\hline MCP-1 (ng/ml) & $143 \cdot 8^{\mathrm{a}}$ & $11 \cdot 7$ & $320 \cdot 3^{\mathrm{b}}$ & $129 \cdot 6$ & $286 \cdot 3^{\mathrm{b}}$ & $123 \cdot 7$ \\
\hline CRP (mg/l) & $223^{\mathrm{a}}$ & 21 & $376^{\mathrm{b}}$ & 41 & $332^{c}$ & 46 \\
\hline IL-6 (pg/ml) & 124.4 & $10 \cdot 6$ & 113.9 & $9 \cdot 3$ & 115.4 & $8 \cdot 3$ \\
\hline $\mathrm{PGE}_{2}(\mathrm{ng} / \mathrm{ml})$ & $2 \cdot 36^{a}$ & 0.85 & $4 \cdot 86^{\mathrm{b}}$ & 1.80 & $5 \cdot 01^{b}$ & 1.66 \\
\hline
\end{tabular}

NC, normal control; DM, diabetes without arginine; DM-Arg, diabetes with arginine.

a,b,c Mean values within a row with unlike superscript letters were significantly different $(P<0.05)$

* Different treatments were analysed by one-way ANOVA using Duncan's test. 
Advanced glycated endproducts receptor expressions in the liver and lungs

Immunocytochemical findings of RAGE-immunoreactive cells with brown cytoplasm and haematoxylin-stained nuclei were seen in liver (Fig. 1(A)-(C)) and lung (Fig. 1(E)-(G)) tissues. In the liver, the immunohistochemical distribution showed that the RAGE-positive cells were frequently observed in the hepatic plates, but not in the sinusoids. It was obvious that the RAGE-positive cells were mostly hepatocytes. In the lungs, the RAGE-positive cells were mostly observed in the wall of alveoli. Immunohistochemical expressions in both the liver and lung tissues showed that the immunoreactive intensities of RAGE were lowest in the NC group (Fig. 1(A) and $(\mathrm{E})$ ). The intensities were lower in the DM-Arg group (Fig. 1(C) and $(\mathrm{G})$ ) than in the DM group (Fig. 1(B) and (F)). Quantification results of RAGE-immunoreactive areas among groups are shown in Fig. 1(D) and (H). The immunoreactive areas of the diabetic groups were significantly larger than those of the NC group. Compared with the DM group, the DM-Arg group had smaller RAGE-immunoreactive areas.
(A)

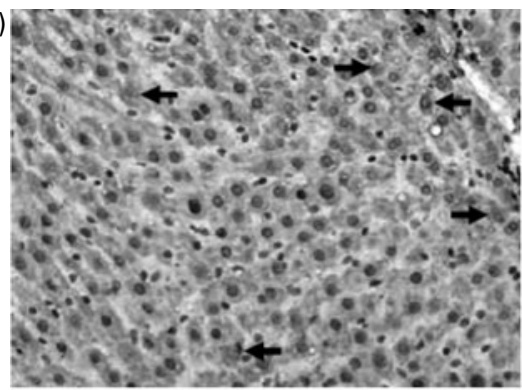

(B)

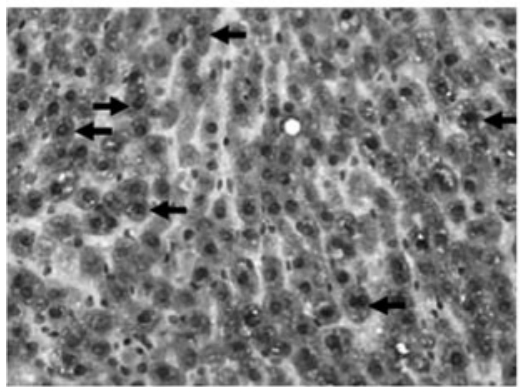

(C)
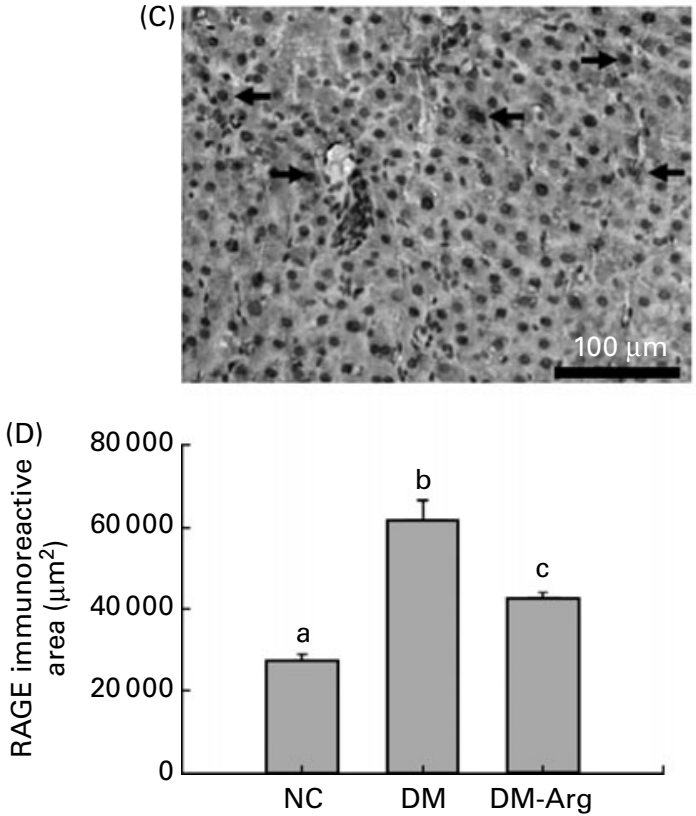
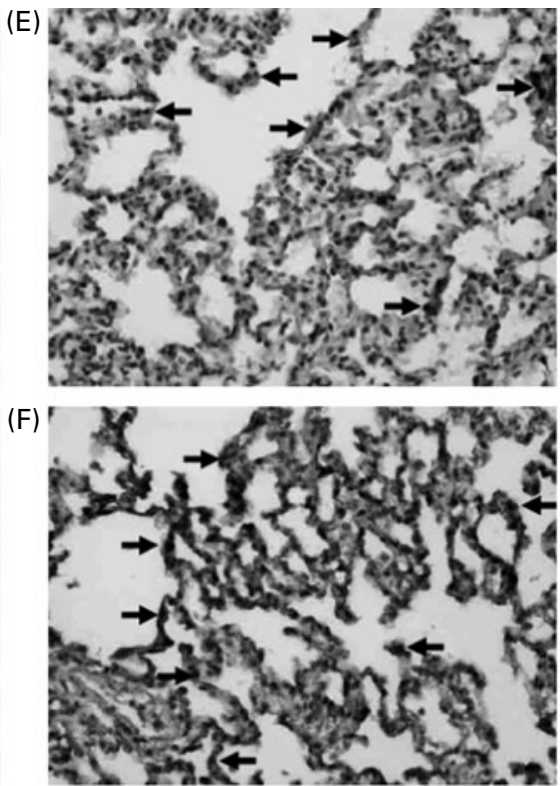

(G)

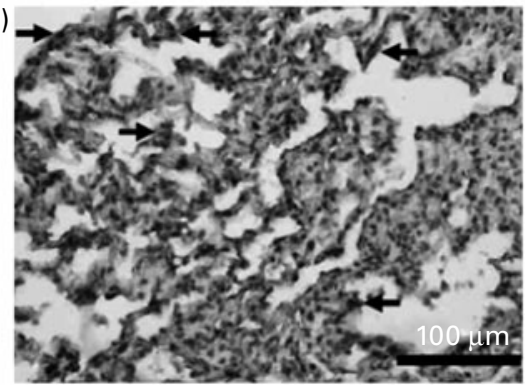

(H)

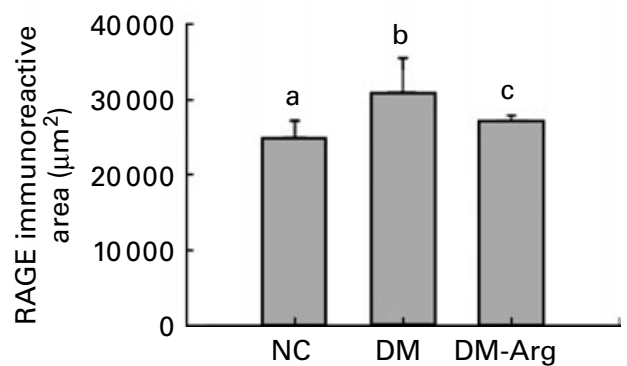

Fig. 1. Receptor of advanced glycation endproducts (RAGE) expression in the liver ( $A, B, C)$ and lungs ( $E, F, G)$ of the normal control (NC) (A, $E$ ), diabetic (DM) $(B, F)$ and diabetic supplemented with arginine (DM-Arg) $(C, G)$ groups. The RAGE-positive cells with brown cytoplasm and haematoxylin-stained nuclei were distributed randomly throughout the tissues, mostly in hepatocytes (A, B, C) and in the alveoli (E, F, G). The RAGE expressions in both of the liver and lung tissues exhibited higher immunoreactive intensities in the DM group (B, F) than in the DM-Arg group (C, G). Scale bars $=100 \mu m$. Quantification of RAGE immunoreactive areas among groups $(n 3)$ in the liver and lungs is shown in $(D)$ and $(H)$, respectively. Areas were assessed with Image-Pro Plus 5.1 (Media Cybernetics, Silver Spring, MD, USA) and were calculated as described in Materials and methods. Values are means, with standard deviations represented by vertical bars. ${ }^{a, b, c}$ Mean values with unlike letters were significantly different $(P<0.05)$. 


\section{Discussion}

In the present study, we used nicotinamide and streptozotocin to achieve a relative deficiency to imitate type 2 diabetes mellitus. Although this is a well-established model of type 2 diabetes mellitus in rodents, the features of insulin resistance and subclinical inflammation secondary to adiposity are not achieved with this method. Results of the present study demonstrated that almost all diabetic rats had hyperglycaemia and hypercholesterolaemia. Arg had no effect on lowering plasma glucose or cholesterol levels. However, TAG and fructosamine contents were reduced when Arg was administered. Accumulating data indicate that there is a link between dyslipidaemia and inflammation; lower plasma lipids may have a favourable effect on attenuating the inflammatory reaction ${ }^{(17)}$. Fructosamines are early glycation adducts which are formed through the condensation of glucose with primary amines. The fructosamine content can be used to quantify advanced glycation endproducts ${ }^{(16)}$. The findings of the present study were consistent with an animal study performed by Liu et al. ${ }^{(18)}$, in which they also found that supplemental dietary Arg decreased fructosamine without influencing fasting glucose levels in diabetic rats. Several reports showed that Arg inhibits in vitro non-enzymic glycation and advanced glycation endproduct formation in serum and tissue proteins from human subjects and animals ${ }^{(19,20)}$. The proposed mechanism was that the amino group of Arg can block an early stage of the Maillard reaction to form non-reactive substituted Amadori products, and with its guanidinium group, Arg may react with dicarbonyls (Amadori breakdown products) which results in fewer advanced glycation endproducts ${ }^{(19)}$. A previous report showed that oral Arg supplementation did not change plasma Arg levels in either control or diabetic rats when compared with their respective groups without $\operatorname{Arg}^{(21)}$. The authors postulated that the protective effect of organ function seen in diabetic rats given Arg was related to less formation of glycosylated products ${ }^{(21)}$.

Advanced glycation endproducts are known to be proinflammatory and pro-oxidant compounds. A previous report revealed that advanced glycation endproducts lead to an induction of reactive oxygen species and promote endothelial expression of IL-6, vascular cell adhesion molecule-1 and MCP- $1^{(22)}$. Prospective epidemiological studies have found that diabetic patients have increased leucocyte counts, proinflammatory cytokines such as IL- 6 and TNF- $\alpha$, and acutephase reactants such as $\mathrm{CRP}^{(23,24)}$. Also, soluble adhesion molecule (intercellular adhesion molecule-1, vascular cell adhesion molecule-1) levels in diabetic patients were significantly higher than those in healthy controls ${ }^{(25)}$. Although $\alpha 2$-macroglobulin is an important acute-phase protein in response to inflammation ${ }^{(26)}$, CRP is considered a potent modulator of inflammatory reaction in rats ${ }^{(27)}$. CRP, IL-6, intercellular adhesion molecule-1, MCP-1 and $\mathrm{PGE}_{2}$ are all markers of inflammation. Adhesion molecules play a key roles in cell-cell and cell-extracellular matrix interactions and are important in the adhesion of leucocytes to activated endothelium ${ }^{(28)}$. Excessive expression of adhesion molecules may induce an inflammatory response and tissue injury ${ }^{(29)}$. MCP-1 is involved in the recruiting peripheral leucocytes ${ }^{(30)}$. $\mathrm{PGE}_{2}$ is a product of arachidonic acid metabolism, which regulates many aspects of the inflammatory and immune systems. Elevated $\mathrm{PGE}_{2}$ production was observed in patients with chronic inflammatory conditions ${ }^{(31)}$. The results of the present study were similar to previous reports in that the inflammatory markers were elevated in the diabetic groups. Although most of the inflammatory markers measured did not differ between the two diabetic groups, we did observe that Arg supplementation reduced CRP levels in diabetes. This finding is consistent with a report by Wells et al. ${ }^{(32)}$, who found an inverse relationship between Arg intake and levels of CRP. In addition, Arg stimulates the secretion of anabolic hormones such as growth hormone, prolactin and insulin-like growth factor$1^{(33)}$, and growth hormone was found to have an effect on decreasing serum $\mathrm{CRP}^{(34)}$. The mechanism of Arg that is responsible for decreasing CRP is unclear and requires further investigation.

RAGE is a member of the immunoglobulin superfamily. RAGE is minimally expressed in normal tissues and the vasculature. However, RAGE is up-regulated when advanced glycation endproduct ligands accumulate. Ligation of RAGE by advanced glycation endproducts results in intracellular signalling which leads to activation of a pro-inflammatory response $^{(35)}$. Engagement of RAGE in the diabetic tissues initiates a vicious cycle of ligand-RAGE perturbation which consequently leads to chronic tissue damage. In the present study, we analysed liver and lung RAGE expressions, because up-regulation of RAGE occurs in tissues and cell types that are critical for immune surveillance including the lungs, liver, vascular endothelium, mononuclear phagocytes, dendritic cells and neurons ${ }^{(36)}$. Lung tissues presented high levels of the RAGE antigen among various organs examined $^{(37)}$. A previous study showed that the kidneys and heart of RAGE-null diabetic mice showed no superimposed injuries. Also, the levels of advanced glycation endproducts in RAGE-null mice were lower than those in RAGE-expressing animals ${ }^{(38)}$. As levels of glycaemia did not differ among groups of diabetic animals, the authors concluded that factors accelerating advanced glycation endproduct formation in the hyperglycaemic environment were modulated by RAGE ${ }^{(38)}$. In the present study, we used immunohistochemistry staining to quantify the expression of RAGE in the liver and lungs of the diabetic rats. We found that RAGE expression is up-regulated in diabetes, whereas Arg administration resulted in lower liver and lung RAGE expressions in the diabetic group. This result is consistent with the lower plasma advanced glycation endproducts and CRP observed in the DM-Arg group. Since the inflammatory mediator CRP and advanced glycation endproduct formation were reduced in the Arg group, this may consequently have reduced the expressions of RAGE in various organs. Also, lower RAGE expressions may down-regulate the production of CRP.

In summary, the present study showed that dietary Arg supplementation resulted in lower advanced glycation endproducts and inflammatory protein production in a diabetic condition. These results are consistent with the immunohistochemical staining which showed that RAGE expressions in the lungs and liver were significantly lower in the Argsupplemented diabetic group. These results suggest that supplemental dietary Arg may decrease advanced glycation 
endproduct-RAGE interactions and consequently reduce tissue damage in rats with type 2 diabetes.

\section{Acknowledgements}

The funding of the present study was supported by a research grant (TMU98-AE1-B07) from Taipei Medical University, Taiwan.

M.-H. P. and K.-H. H contributed to the concept of the study and did the data analysis. S.-L.Y. designed the study and prepared the manuscript, and C.-H. W interpreted the data. All authors read and approved the final submitted manuscript.

The authors are not employees or consultants associated with any commercial companies, and there were no conflicts of interest in the present study.

\section{References}

1. Department of Health, Taiwan (2008) Top 10 leading causes of death in Taiwan. www.doh.gov.tw (accessed June 2009).

2. Goh SY \& Cooper ME (2008) Clinical review: the role of advanced glycation end products in progression and complications of diabetes. J Clin Endocrinol Metab 93, $1143-1152$.

3. Bierhaus A, Hofmann MA, Ziegler R, et al. (1998) AGEs and their interaction with AGE-receptors in vascular disease and diabetes mellitus. I. The AGE concept. Cardiovasc Res 37, 586-600.

4. Bierhaus A, Humpert PM, Morcos M, et al. (2005) Understanding RAGE, the receptor for advanced glycation end products. $J$ Mol Med 83, 876-886.

5. Hnia K, Gayraud J, Hugon G, et al. (2008) L-Arginine decreases inflammation and modulates the nuclear factor- $\mathrm{\kappa B} /$ matrix metalloproteinase cascade in $\mathrm{mdx}$ muscle fibers. Am J Pathol 172, 1509-1519.

6. Nieves C Jr \& Langkamp-Henken B (2002) Arginine and immunity: a unique perspective. Biomed Pharmacother 56, 471-482.

7. Gianotti L, Alexander JW, Pyles T, et al. (1993) Argininesupplemented diets improve survival in gut-derived sepsis and peritonitis by modulating bacterial clearance. The role of nitric oxide. Ann Surg 217, 644-653.

8. Evoy D, Lieberman MD, Fahey TJ III, et al. (1998) Immunonutrition: the role of arginine. Nutrition 14, 611-617.

9. Casas-Rodera P, Gomez-Candela C, Benitez S, et al. (2008) Immunoenhanced enteral nutrition formulas in head and neck cancer surgery: a prospective, randomized clinical trial. Nutr Hosp 23, 105-110.

10. Martina V, Masha A, Gigliardi VR, et al. (2008) Long-term $N$-acetylcysteine and L-arginine administration reduces endothelial activation and systolic blood pressure in hypertensive patients with type 2 diabetes. Diabet Care 31, 940-944.

11. Lucotti P, Setola E, Monti LD, et al. (2006) Beneficial effects of a long-term oral L-arginine treatment added to a hypocaloric diet and exercise training program in obese, insulin-resistant type 2 diabetic patients. Am J Physiol Endocrinol Metab 291, E906-E912.

12. Masiello P, Broca C, Gross R, et al. (1998) Experimental NIDDM: development of a new model in adult rats administered streptozotocin and nicotinamide. Diabetes 47, 224-229.

13. Masiello P, Novelli M, Fierabracci V, et al. (1990) Protection by 3 -aminobenzamide and nicotinamide against streptozotocin-induced $\beta$-cell toxicity in vivo and in vitro. Res Commun Chem Pathol Pharmacol 69, 17-32.
14. Yeh CL, Pai MH, Li CC, et al. (2009) Effect of arginine on angiogenesis induced by human colon cancer: in vitro and in vivo studies. $J$ Nutr Biochem (Epublication ahead of print version 14 May 2009).

15. Shang HF, Wang YY, Lai YN, et al. (2004) Effects of arginine supplementation on mucosal immunity in rats with septic peritonitis. Clin Nutr 23, 561-569.

16. Chung HF, Lees H \& Gutman SI (1988) Effect of nitroblue tetrazolium concentration on the fructosamine assay for quantifying glycated protein. Clin Chem 34, 2106-2111.

17. Libby P, Ridker PM \& Maseri A (2002) Inflammation and atherosclerosis. Circulation 105, 1135-1143.

18. Liu CT, Chen KM, Lee SH, et al. (2005) Effect of supplemental L-arginine on the function of $\mathrm{T}$ lymphocytes and the formation of advanced glycosylated end products in rats with streptozotocin-induced diabetes. Nutrition 21, $615-623$.

19. Servetnick DA, Bryant D, Wells-Knecht KJ, et al. (1996) L-Arginine inhibits in vitro nonenzymatic glycation and advanced glycosylated end product formation of human serum albumin. Amino Acids 11, 69-81.

20. Menzel EJ \& Reihsner R (1996) Comparison of the effect of different inhibitors of the non-enzymatic glycation of rat tail tendons and bovine serum albumin. Ann Clin Biochem 33, $241-248$.

21. Reyes AA, Karl IE, Kissane J, et al. (1993) L-Arginine administration prevents glomerular hyperfiltration and decreases proteinuria in diabetic rats. J Am Soc Nephrol 4, $1039-1045$.

22. Zhang L, Zalewski A, Liu Y, et al. (2003) Diabetes-induced oxidative stress and low-grade inflammation in porcine coronary arteries. Circulation 108, 472-478.

23. Schmidt MI, Duncan BB, Sharrett AR, et al. (1999) Markers of inflammation and prediction of diabetes mellitus in adults (atherosclerosis risk in communities study): a cohort study. Lancet 353, 1649-1652.

24. Pradhan AD, Manson JE, Rifai N, et al. (2001) C-reactive protein, interleukin-6, and risk of developing type 2 diabetes mellitus. JAMA 286, 327-334.

25. Glowinska B, Urban M, Peczynska J, et al. (2005) Soluble adhesion molecules (sICAM-1, sVCAM-1) and selectins ( $\mathrm{sE}$ selectin, sP selectin, sL selectin) levels in children and adolescents with obesity, hypertension, and diabetes. Metabolism 54, 1020-1026.

26. Mirjana M, Silva D, Goran P, et al. (2009) The acute-phase protein $\alpha 2$-macroglobulin plays an important role in radioprotection in the rat. Shock 31, 607-614.

27. Sun H, Lu X, Wu S, et al. (2009) The effects of C-reactive protein, interleukin- 6 , and tumor necrosis factor- $\alpha$ in rat allograft adventitial inflammation and allograft arteriosclerosis. Transplant Proc 41, 3909-3912.

28. Jang Y, Lincoff AM, Plow EF, et al. (1994) Cell adhesion molecules in coronary artery disease. J Am Coll Cardiol 24, $1591-1601$.

29. Albelda SM, Smith CW \& Ward PA (1994) Adhesion molecules and inflammatory injury. FASEB $J$ 8, 504-512.

30. Matsukawa A, Hogaboam CM, Lukacs NW, et al. (1999) Endogenous monocyte chemoattractant protein-1 protect mice in a model of acute septic peritonitis: cross-talk between MCP-1 and leukotriene $\mathrm{B}_{4}$. J Immunol 163, 6148-6154.

31. Calder PC (1997) n-3 Polyunsaturated fatty acids as pharmacologic agents: a fishy tale? Nutrition 13, 1002-1004.

32. Wells BJ, Mainous AG III \& Everett CJ (2005) Association between dietary arginine and C-reactive protein. Nutrition 21, $125-130$

33. Roth E (2007) Immune and cell modulation by amino acids. Clin Nutr 26, 535-544. 
34. Graham MR, Baker JS, Evans P, et al. (2007) Evidence for a decrease in cardiovascular risk factors following recombinant growth hormone administration in abstinent anabolicandrogenic steroid users. Growth Horm IGF Res 17, 201-209.

35. Yan SF, Ramasamy R \& Schmidt AM (2009) Receptor for AGE (RAGE) and its ligands cast into leading roles in diabetes and the inflammatory response. $\mathrm{J} \mathrm{Mol} \mathrm{Med} \mathrm{87,}$ $235-247$.
36. Lin L, Park S \& Lakatta EG (2009) RAGE signaling in inflammation and arterial aging. Front Biosci 14, 1403-1413.

37. Brett J, Schmidt AM, Yan SD, et al. (1993) Survey of the distribution of a newly characterized receptor for advanced glycation end products in tissues. Am J Pathol 143, 1699-1712.

38. Bucciarelli LG, Ananthakrishnan R, Hwang YC, et al. (2008) RAGE and modulation of ischemic injury in the diabetic myocardium. Diabetes 57, 1941-1951. 